\title{
Variants in CPT1A, FADS1, and FADS2 are associated with higher levels of estimated plasma and erythrocyte delta-5 desaturases in Alaskan Eskimos
}

\author{
V. Saroja Voruganti ${ }^{1}$ *, Paul B. Higgins ${ }^{2}$, Sven O. E. Ebbesson ${ }^{3}$, John Kennish ${ }^{4}$, Harald H. H. Göring ${ }^{1}$, \\ Karin Haack ${ }^{1}$, Sandra Laston ${ }^{1}$, Eugene Drigalenko ${ }^{1}$, Charlotte R. Wenger ${ }^{1}$, William S. Harris ${ }^{5}$, \\ Richard R. Fabsitz ${ }^{6}$, Richard B. Devereux ${ }^{7}$, Jean W. MacCluer ${ }^{1}$, Joanne E. Curran ${ }^{1}$, Melanie A. Carless ${ }^{1}$, \\ Matthew P. Johnson ${ }^{1}$, Eric K. Moses ${ }^{1,8}$, John Blangero ${ }^{1}$, Jason G. Umans ${ }^{9}$, Barbara V. Howard ${ }^{9}$, \\ Shelley A. Cole ${ }^{1}$ and Anthony Gean Comuzzie ${ }^{1}$
}

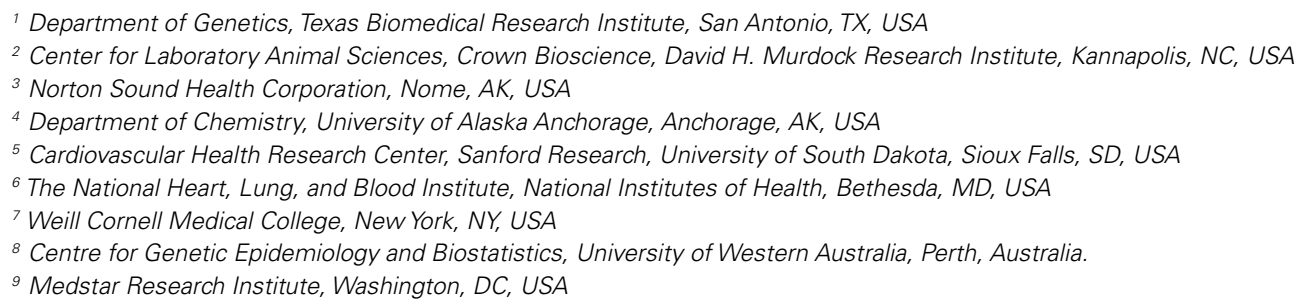

\section{Edited by:}

Karen T. Cuenco, University of Pittsburgh, USA

\section{Reviewed by:}

Yiran Guo, Children's Hospital of Philadelphia, USA

Maria J. Jesus Moreno-Aliaga, University of Navarra, Spain

*Correspondence:

V. Saroja Voruganti, Department of Genetics, Texas Biomedical Research Institute, P.O. Box 760549, San Antonio, TX 78245-0549, USA. e-mail:saroja@txbiomedgenetics.org
The delta- 5 and delta- 6 desaturases (D5D and D6D), encoded by fatty acid desaturase 1 (FADS1) and 2 (FADS2) genes, respectively, are rate-limiting enzymes in the metabolism of $\omega-3$ and $\omega-6$ fatty acids. The objective of this study was to identify genes influencing variation in estimated D5D and D6D activities in plasma and erythrocytes in Alaskan Eskimos $(n=761)$ participating in the genetics of coronary artery disease in Alaska Natives (GOCADAN) study. Desaturase activity was estimated by product: precursor ratio of polyunsaturated fatty acids. We found evidence of linkage for estimated erythrocyte D5D (eD5D) on chromosome 11q12-q13 (logarithm of odds score $=3.5$ ). The confidence interval contains candidate genes FADS1, FADS2, 7-dehydrocholesterol reductase (DHCR7), and carnitine palmitoyl transferase $1 \mathrm{~A}$, liver (CPT1A). Measured genotype analysis found association between CPT1A, FADS1, and FADS2 single-nucleotide polymorphisms (SNPs) and estimated eD5D activity ( $p$-values between $10^{-28}$ and $10^{-5}$ ). A Bayesian quantitative trait nucleotide analysis showed that rs3019594 in CPT1A, rs174541 in FADS1, and rs174568 in FADS2 had posterior probabilities $>0.8$, thereby demonstrating significant statistical support for a functional effect on eD5D activity. Highly significant associations of FADS1, FADS2, and CPT1A transcripts with their respective SNPs ( $p$-values between $10^{-75}$ and $10^{-7}$ ) in Mexican Americans of the San Antonio Family Heart Study corroborated our results. These findings strongly suggest a functional role for FADS1, FADS2, and CPT1A SNPs in the variation in eD5D activity.

Keywords: essential fatty acids, single-nucleotide polymorphisms, bayesian quantitative trait nucleotide analysis

\section{INTRODUCTION}

Essential fatty acids are important constituents of all cell membranes and influence both inflammatory and atherosclerotic processes. The essential fatty acids cis-linoleic acid (LA;18: $2 \omega-6)$ and $\alpha$-linolenic acid (ALA; 18:3 $\omega$-3) are metabolized to arachidonic acid (AA; 20:4 $\omega-6)$ and eicosapentaenoic acid (EPA; 20:5 $\omega-3)$, respectively, by delta-5 (D5D) and delta-6 (D6D) desaturases (Das, 2007; Figure 1). Since most of the products of the arachidonic acid pathway have pro-inflammatory effects, increased D5D and D6D activity in the $\omega-6$ pathway may promote systemic inflammation and increase the risk of atherosclerosis (Das, 2007; Allayee et al., 2009; Martinelli et al., 2009). Conversely, increased activities of these desaturases could stimulate the synthesis of
EPA (which has anti-inflammatory properties) from ALA, counteracting the effects of AA synthesis. However, both EPA and AA are synthesized at very low rates in vivo (Harris et al., 2009; Mozaffarian et al., 2010).

Diet, metabolism, and genetic variation influence the fatty acid composition of the plasma lipids and cell membranes. The variation in plasma and dietary fatty acids, particularly polyunsaturated fatty acids (PUFAs), has been shown to be under considerable genetic influence (Cai et al., 2004; Tanaka et al., 2009; Voruganti et al., 2010). Recent genome-wide association studies (GWAS) have shown a strong association between PUFAs and single-nucleotide polymorphisms (SNPs) in fatty acid desaturase 1 and 2 (FADS1 and FADS2) genes, which code for D5D and D6D, 


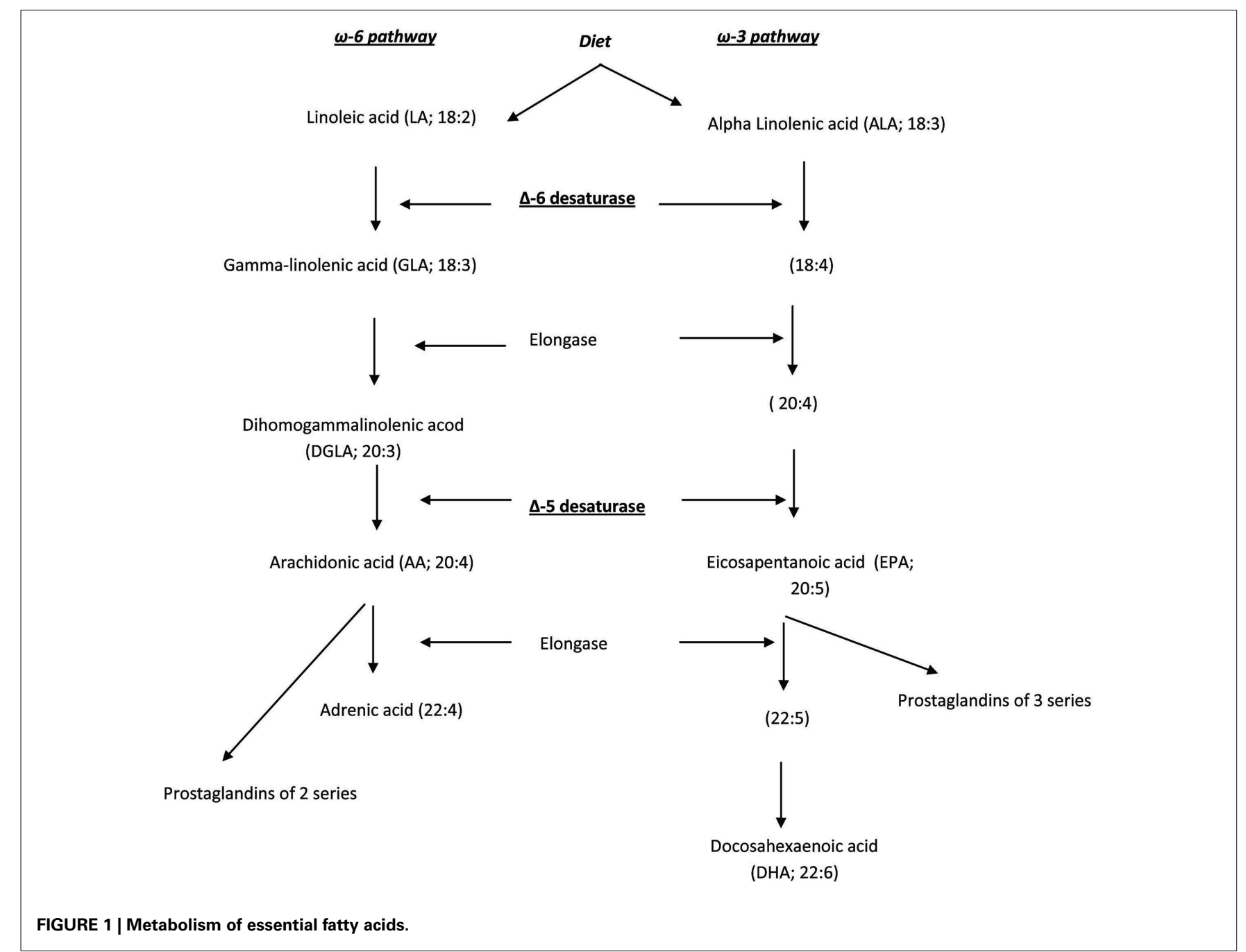

respectively (Tanaka et al., 2009). These associations have been further confirmed by candidate gene studies in various populations (Schaeffer et al., 2006; Malerba et al., 2008; Martinelli et al., 2008; Lattka et al., 2009, 2010; Rzehak et al., 2009; Bokor et al., 2010; Glaser et al., 2010; Mathias et al., 2010; Merino et al., 2011). In addition, these GWAS have found association between FADS1 and FADS2 SNPs and cardiovascular disease (CVD) risk factors (Tanaka et al., 2009). Both genes are located on chromosome 11q12-q13.1. Genome-wide linkage and association studies have been conducted with plasma fatty acids (Schaeffer et al., 2006; Malerba et al., 2008; Martinelli et al., 2008; Lattka et al., 2009, 2010; Rzehak et al., 2009; Bokor et al., 2010; Glaser et al., 2010; Mathias et al., 2010; Voruganti et al., 2010; Merino et al., 2011), but few studies have investigated genetic influences on estimated desaturase activities in blood (Martinelli et al., 2008; Bokor et al., 2010; Mathias et al., 2010; Merino et al., 2011).

Westernized diets are characterized by higher amounts of $\omega-6$ PUFA more inclined to promote pro-inflammatory activities in contrast to $\omega$-3 PUFA (Allayee et al., 2009; Martinelli et al., 2009). The Alaskan Eskimo population, which is transitioning away from an $\omega-3$ fatty acid-rich diet to a relatively westernized diet, is thus at a higher risk for systemic inflammation and atherosclerosis.
Given these facts and the importance of D5D and D6D in essential fatty acid metabolism and inflammation, we investigated the genetic influence on estimated D5D and D6D desaturase activities. Herein we present the results of a genome-wide scan and candidate SNP analyses for estimated D5D and D6D activities in Alaskan Eskimos participating in the genetics of coronary artery disease in Alaska Natives (GOCADAN) study.

\section{MATERIALS AND METHODS STUDY DESIGN}

In the GOCADAN study, 1214 individuals (all 18 years of age and older) were recruited from villages in the Norton Sound region on the northwestern coast of Alaska. Details of the study recruitment, design, and methods have been reported by Howard et al. (2005); Ebbesson et al. (2006). The Institutional Review Boards from all participating institutions approved this study and informed consent was obtained from all participants.

\section{DEMOGRAPHIC AND PHENOTYPIC DATA}

Demographic and genealogical data collected during the interviews included name, gender, date and place of birth, current home of the participant and his or her spouse, and first-degree relatives of 
all household members. Anthropometric measurements, height, weight, and waist and hip circumference, were measured by standardized procedures. Body mass index (BMI) was calculated by dividing weight (kilograms) by height (meters) squared. Body fat was measured using a Quantum II bioelectrical body composition analyzer (RJL Systems, Clinton Township, MI). Triglycerides, highdensity lipoprotein (HDL), low-density lipoprotein (LDL), and total cholesterol were measured using an auto analyzer (Hitachi 717, Amposta, Spain). Heart rate was measured via computerized ECG (electrocardiograph; GE Systems MAC 1200), with the participant at rest. Heart rate was calculated using the mean interval between QRS complexes during a 10-s period. Apolipoprotein A1 (ApoA1) was analyzed by immunoprecipitation assay.

Of the 1214 individuals participating in the study, fatty acid concentrations and desaturases data were available for 761 individuals ( $>35$ years of age). Total plasma and erythrocyte fatty acid concentrations were estimated following the methods previously described by Ebbesson et al. (1999) and Ebbesson et al. (2010), respectively. In short, analysis of the composition of fatty acids was performed on a Hewlett Packard 5890 gas chromatograph equipped with a $60 \mathrm{~m} \times 0.25 \mathrm{~mm}$ SP330 capillary column chemically bonded with $0.25 \mu \mathrm{m}$ liquid phase and a flame ionization detector. Structural confirmation was determined by mass spectrometry using a Varian 2000 ion trap detector. Each fatty acid measure was computed using a regression equation in which the ratio of the area of each fatty acid peak to the internal standard was plotted against the weight ratio of the fatty acid and the internal standard. All fatty acids are expressed as percentages $(\mathrm{mol} / 100 \mathrm{ml}$ of total fatty acids).

Desaturases in plasma and erythrocytes were estimated by product-precursor ratio of PUFAs (Warensjö et al., 2008).

$$
\mathrm{D} 5 \mathrm{D}=[(20: 4 \omega-6) /(20: 3 \omega-6)]
$$$$
\mathrm{D} 6 \mathrm{D}=[(18: 3 \omega-6) /(18: 2 \omega-6)]
$$

\section{GENOTYPE DATA}

For each of the participants, 400 short tandem repeat (STR) markers (spaced at an average interval of $10 \mathrm{cM}$ throughout the genome) were amplified from genomic DNA in separate polymerase chain reactions (PCR) using fluorescently labeled primer pairs (ABI PRISM Linkage Mapping Set MD 10 Version 2, Applied Biosystems, Foster City, CA, USA). Pedigree and Mendelian errors were detected and corrected utilizing the software pedigree relationship statistical tests (PREST) and SIMWALK (Sobel and Lange, 1996). Multipoint identity-by-descent (IBD) matrices for genome-wide linkage analyses were calculated using the linkage analysis package LOKI (Heath, 1997). The chromosomal map (Haldane) used in these computations was based on marker locations reported by DeCode genetics (Kong et al., 2002).

\section{SNP SELECTION AND GENOTYPING}

We genotyped 324 SNPs clustered in the candidate genes within the one-logarithm of odds (LOD) support interval of our quantitative trait locus (QTL) region (26 in FADS1, 100 in FADS2, 149 in carnitine palmitoyl transferase 1A, liver (CPT1A), and 49 in 7-dehydrocholesterol reductase (DHCR7)). These SNPs were selected to be within $2 \mathrm{~kb}$ upstream to $1 \mathrm{~kb}$ downstream of each gene and were previously reported to be polymorphic in both
Asian and European populations. Of the 324 SNPs included in data collection, 34 failed and 78 were not polymorphic in our study population, leaving 212 polymorphic markers for analysis $(F A D S 1=16, F A D S 2=68, D H C R 7=30$, and $C P T 1 A=98)$. SNPs were typed using the multiplex VeraCode technology from Illumina according to the manufacturer's protocol (Illumina, San Diego, CA, USA).

Details of the technology are given in Voruganti et al. (2010). Briefly, the technology is based on allele-specific primer extension. Genomic DNA (250 ng) was chemically activated, labeled with biotin, and then hybridized to a pool of locus-specific oligos. After primer extension and ligation, a PCR reaction was performed using a flurorescent-labeled primers, followed by hybridization to VeraCode beads. The beads were then scanned on a BeadXpress reader using the VeraScan software (Illumina). Genotype calls were generated by analyzing the raw data using the software BeadStudio (Illumina).

\section{STATISTICAL ANALYSES Univariate genetic analysis}

A variance components decomposition method was used to estimate heritability and detect linkage to chromosomal locations affecting variation in estimated plasma and erythrocyte desaturase activities via genome-wide linkage analysis. This method was implemented in the software program SOLAR and has been described in detail elsewhere (Blangero and Almasy, 1997; Almasy and Blangero, 1998). Before conducting genetic analyses, distributional properties of all traits were evaluated. All values greater than four standard deviations from the mean were removed and the remaining traits were transformed by inverse normalization to meet assumptions of normality. All analyses were conducted adjusting for age, sex, diabetes status, and use of lipid-lowering medication.

\section{Measured genotype analysis (MGA)}

Genotype frequencies for each SNP were calculated using a maximum-likelihood estimation method and were tested for departures from Hardy-Weinberg equilibrium. Estimates of linkage disequilibrium (LD) between SNPs were determined by calculating pair-wise $\mathrm{D}^{\prime}$ and $r^{2}$ statistics. As a first step in investigating the association between the SNPs in candidate genes and variation in estimated desaturase activities, we employed MGA implemented in SOLAR (Boerwinkle et al., 1986). This approach extends the classical variance components-based biometrical model to account for both the random effects of kinship and the main effects of SNP genotypes. For each SNP, we compared this saturated model with a null model in which the main effect of the SNP is constrained to zero. The test statistic, twice the difference in $\log _{e}$ (likelihood) between the saturated model and the SNP-specific null, has a chi-square distribution with one degree of freedom.

\section{Bayesian quantitative trait nucleotide analysis}

The measured genotype analysis described above was used to assess association between the trait and each SNP, analyzing one SNP at a time. However, multiple functional variants will often exist within a chromosomal locus, and joint analysis of multiple variants may be more powerful to detect their effects and to establish which of 
the many polymorphisms within a region are the most likely to be functional. Blangero et al. (2005) developed the Bayesian quantitative trait nucleotide (BQTN) approach, which is essentially a Bayesian method based on an underlying measured genotype model that permits joint analysis of multiple variants. The BQTN analysis was implemented in SOLAR to identify the functional SNPs that are statistically most likely to be associated with a phenotype. This method has been described in detail elsewhere (Blangero et al., 2005).

\section{Conditional linkage analysis}

Linkage analysis (Sun et al., 2002) for estimated erythrocyte D5D was performed conditional on SNPs that showed statistical evidence of functionality. If the SNP or SNPs account for the linkage signal completely, the LOD score will be reduced to zero.

\section{SNP GENOTYPING AND IDENTIFICATION AND ANALYSIS OF EXPRESSION TRANSCRIPTS IN MEXICAN AMERICANS}

The SNPs selected for replication were part of the GWAS conducted in the San Antonio Family Heart Study (SAFHS). The SAFHS, a study initiated in 1991, focuses on identifying genetic and environmental risk factors for CVD in Mexican Americans without regard to disease status. GWAS was conducted with SNPs obtained using Illumina HumanHap550 genotyping BeadChip (Illumina Inc., San Diego, CA). Lymphocyte expression data were available for 1240 individuals participating in the SAFHS. However, data on both transcripts and SNPs were available for 1189 individuals. Details of identification and analysis of transcripts are given in Göring et al. (2007). Measured genotype analysis was conducted to analyze the association of FADS1, FADS2, and CPT1A SNPs and their cis-regulated transcripts and CVD-related traits in Mexican Americans.

\section{RESULTS \\ ESTIMATION OF HERITABILITY AND LOCALIZATION OF OTLS}

Erythrocyte and plasma D5D and D6D activities were significantly heritable (Table 1), with values ranging between 0.50 and 0.90 . Genome-wide linkage scans for D5D and D6D revealed a QTL with strong linkage on chromosome 11 for estimated erythrocyte D5D (Figure 1). This QTL was localized to chromosome 11q12-q13 near marker D11S987 with a LOD score of 3.5 (Table 1, Figure 2). The one-LOD support interval for this QTL spanned approximately $20 \mathrm{cM}$ between markers D11S4191 and D11S1314. This interval contains promising candidate genes including the
FADS1 gene that codes for D5D. Other candidate genes in this interval are FADS2, DHCR7, and CPT1A.

\section{MEASURED GENOTYPE ANALYSIS}

Linkage disequilibrium patterns of these SNPs are shown in Figure S1 in Supplementary Material. All of the SNPs were tested for association with estimated erythrocyte and plasma D5D and D6D and their individual components. Polymorphisms in FADS1, FADS2, and CPT1A showed strong association with erythrocyte and plasma D5D and D6D (Figure 3). Estimated erythrocyte D5D and its components 20:3 $\omega-6$ and 20:4 $\omega-6$ showed the strongest association, with $p$-values ranging from $10^{-28}$ to $10^{-5}$ (Table 2). Similarly, estimated plasma D5D and its components, used to estimate the ratio, showed significant associations with SNPs in the three genes ( $p$-values $10^{-39}$ to $10^{-16}$ ). However, the association of plasma 20:3 $\omega-6$ with these SNPs was not significant after adjusting for multiple testing. For estimated plasma D6D, only the association with SNPs in the FADS1 and FADS2 genes was significant, whereas association with erythrocyte D6D was significant for SNPs in all three genes (Table 3). Detailed results of the association with estimated plasma and erythrocyte D5D and D6D activities and their individual components are shown in Tables S1 and S2 in Supplementary Material.

These SNPs were also tested for their association with cardiovascular risk factors, heart rate, plaque, triglycerides, HDL, LDL, and total cholesterol. SNPs in all three genes were associated with heart rate and HDL cholesterol (Table 4). However, after adjusting for multiple testing, only the association with heart rate remained significant. Individuals with minor alleles of these genes had a lower heart rate.

\section{BOTN ANALYSIS}

Because BQTN analysis is designed to identify SNPs that are most likely to have a functional effect on a trait and it is robust to multiple testing, (Boerwinkle et al., 1986) the SNPs were next tested by BQTN analysis. In the current analyses, SNPs were utilized after removing SNPs that were in LD (>90\%) with other SNPs. One variant from each gene showed a posterior probability greater than 0.8. SNPs rs174541 from FADS1 and rs174568 from FADS2 showed posterior probabilities of 1 , indicating a strong probability of having a functional effect on erythrocyte D5D. However, with plasma D5D, rs3019594 of CPT1A was the only SNP to show a posterior probability of 1 . The genotype-specific phenotype means show minor alleles of all three SNPs to be associated with higher

Table 1 | Heritabilities and linkage results for D5D and D6D*.

\begin{tabular}{|c|c|c|c|c|c|c|}
\hline Trait & Mean (SD) & $h^{2}$ (SE) & $p$-Value & Chr & Location (cM) & LOD score \\
\hline \multicolumn{7}{|c|}{ PLASMA } \\
\hline D5D & $0.683(0.30)$ & $0.502(0.15)$ & 0.00036 & 3 & 97 & 1.615 \\
\hline \multicolumn{7}{|c|}{ ERYTHROCYTE } \\
\hline D5D & $8.015(2.6)$ & $0.544(0.11)$ & 0.0000003 & 11 & 82 & 3.53 \\
\hline
\end{tabular}

${ }^{*}$ Age, sex, diabetes status, and lipid medication were used as covariates. 
levels of erythrocyte and plasma D5D (Table 5). None of these SNPs showed posterior probabilities greater than 0.5 for estimated plasma or erythrocyte D6D activities.

\section{CONDITIONAL LINKAGE}

A linkage scan conditional on the three SNPs, which showed posterior probability of 0.8 and greater, decreased the LOD score for erythrocyte D5D significantly from 3.5 to 1.5 for CPT1A SNP and 1.9 for FADS1 and FADS2 SNPs (Figure 4).

\section{EXAMINATION OF CIS-REGULATED FADS1, FADS2, AND CPT1A TRANSCRIPTS IN MEXICAN AMERICANS}

In a previous study by Göring et al. (2007), linkage analysis was conducted to identify genetic factors that affect the variation in

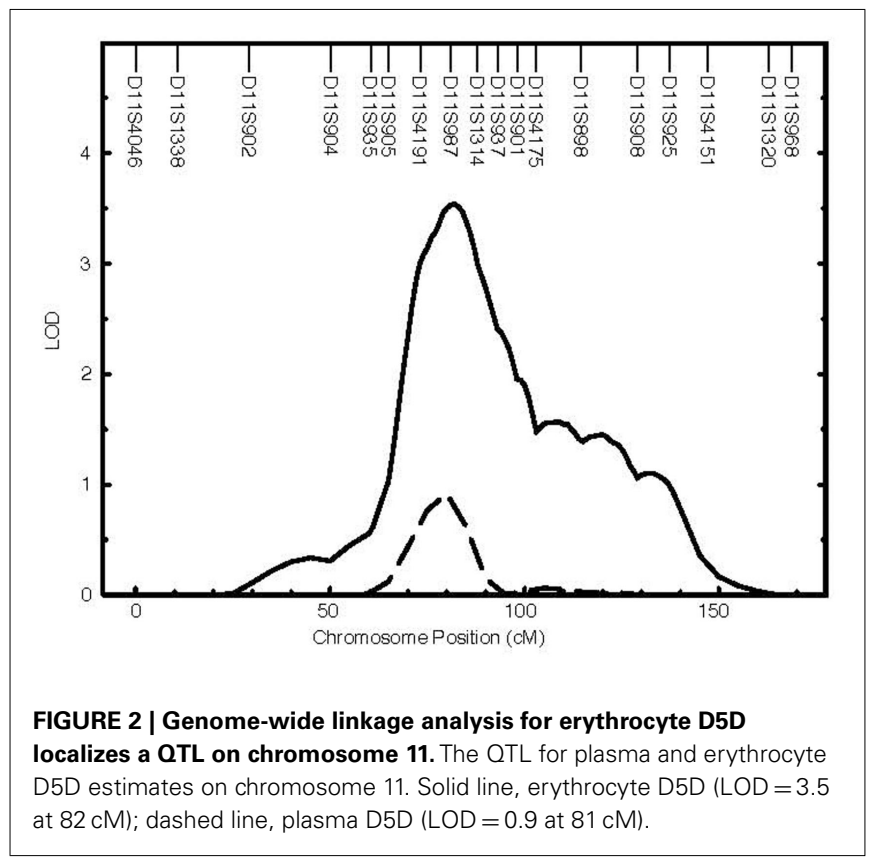

A CPTIA

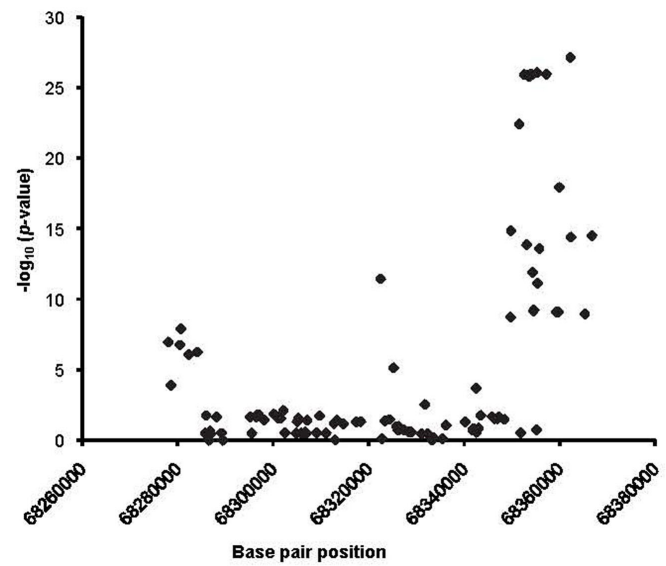

the levels of gene expression. The study identified a number of known cis-regulated transcripts with high-magnitude LOD scores. Included among these, FADS1, FADS2, and CPT1A transcript levels were found to be strongly associated with SNPs located nearby (cis) their coding locus, with $p$-values ranging from $10^{-75}$ to $10^{-7}$. Just as in the GOCADAN study, these SNPs were also tested for their association with cardiovascular risk factors, heart rate, plaque, triglycerides, HDL, LDL, and total cholesterol in the SAFHS, a large family-based study of genetics of coronary artery disease in Mexican Americans. Nominal associations were obtained between the SNPs and ApoA1, and HDL, LDL, total cholesterol and heart rate in the SAFHS (Table 6).

\section{DISCUSSION}

The key finding of this study is the strong statistical support for functional effects of FADS1, FADS2, and CPT1A SNPs on the variation in estimated erythrocyte and plasma D5D, a rate-limiting enzyme in the conversion of essential fatty acids to AA (20:4 $\omega$ $6)$ and EPA (20; $5 \omega-3)$. High heritability estimates for estimated plasma and erythrocyte D5D and D6D activities observed in this study show that there is a significant genetic influence on their variation. Although no study has so far computed heritabilities for desaturase activities, heritabilities of their individual components have been estimated and have been found to be lower than our desaturase estimates (Tanaka et al., 2009; Voruganti et al., 2010). Similarly, no linkage studies have been conducted for estimated desaturase activities. A genome-wide scan conducted for plasma and erythrocyte D5D and D6D activities in the current study showed evidence of a highly significant QTL on chromosome 11q12-q13 for estimated erythrocyte D5D. This region has four potential candidate genes, FADS1, FADS2, CPT1A, and DHCR7. To follow up on our QTL, we genotyped SNPs in the four potential candidate genes and conducted measured genotype-based association analyses with estimated plasma and erythrocyte D5D and D6D. Desaturases are key enzymes facilitating the introduction of double bonds between carbon atoms of fatty acyl chains in

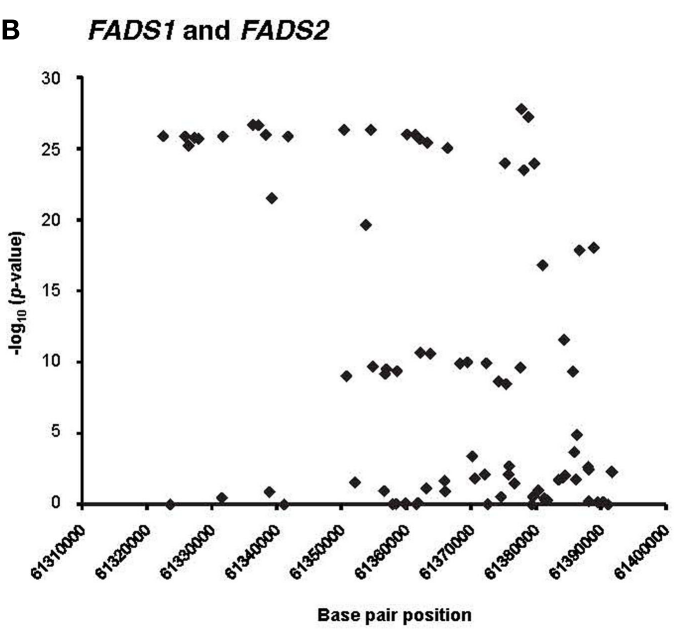

FIGURE 3 | Manhattan plots showing the association of SNPs on CPT1A and FADS1 and FADS2 with erythrocyte D5D. (A) CPT1A and (B) FADS1and FADS2. 
Table 2 | Measured genotype association results for SNPs most significantly associated with D5D.

\begin{tabular}{|c|c|c|c|c|c|c|c|c|}
\hline \multirow[t]{3}{*}{ Gene } & \multirow[t]{3}{*}{ SNP } & \multirow[t]{3}{*}{ Minor allele (frequency) } & \multicolumn{6}{|c|}{ MGA ( $p$-value $)^{*}$} \\
\hline & & & \multicolumn{3}{|c|}{ RBC } & \multicolumn{3}{|c|}{ Plasma } \\
\hline & & & $20: 4 \omega-6$ & $20: 3 \omega-6$ & D5D & $20: 4 \omega-6$ & $20: 3 \omega-6$ & D5D \\
\hline \multirow[t]{3}{*}{ FADS1 } & rs174541 & $A(0.113)$ & $2.2 \times 10^{-26}$ & $1.8 \times 10^{-5}$ & $8.1 \times 10^{-28}$ & $1.2 \times 10^{-28}$ & $8.8 \times 10^{-3}$ & $8.5 \times 10^{-16}$ \\
\hline & rs174546 & $\mathrm{G}(0.114)$ & $1.5 \times 10^{-24}$ & $2.7 \times 10^{-5}$ & $4.3 \times 10^{-26}$ & $3.6 \times 10^{-28}$ & 0.009 & $2.4 \times 10^{-15}$ \\
\hline & rs174556 & $\mathrm{G}(0.122)$ & $2.2 \times 10^{-26}$ & $1.1 \times 10^{-26}$ & $8.1 \times 10^{-28}$ & $1.3 \times 10^{-28}$ & 0.009 & $8.9 \times 10^{-16}$ \\
\hline \multirow[t]{6}{*}{ FADS2 } & rs1535 & $A(0.116)$ & $1.9 \times 10^{-25}$ & $1.5 \times 10^{-5}$ & $2.5 \times 10^{-27}$ & $2.3 \times 10^{-29}$ & 0.012 & $2.8 \times 10^{-16}$ \\
\hline & rs174568 & $\mathrm{G}(0.115)$ & $1.1 \times 10^{-25}$ & $9.5 \times 10^{-6}$ & $2.2 \times 10^{-27}$ & $7.7 \times 10^{-30}$ & 0.040 & $1.7 \times 10^{-24}$ \\
\hline & rs174570 & $\mathrm{G}(0.161)$ & $1.3 \times 10^{-28}$ & 0.009 & $8.2 \times 10^{-21}$ & $1.4 \times 10^{-34}$ & 0.015 & $1.2 \times 10^{-19}$ \\
\hline & rs174576 & $C(0.116)$ & $7.1 \times 10^{-25}$ & $8.5 \times 10^{-6}$ & $5.1 \times 10^{-27}$ & $2.0 \times 10^{-28}$ & 0.012 & $2.3 \times 10^{-16}$ \\
\hline & rs174579 & $\mathrm{G}(0.426)$ & 0.0004 & $9.9 \times 10^{-7}$ & $1.9 \times 10^{-11}$ & $6.1 \times 10^{-9}$ & 0.866 & $5.7 \times 10^{-6}$ \\
\hline & rs968567 & $A(0.038)$ & 0.096 & 0.0003 & 0.032 & 0.023 & 0.625 & 0.042 \\
\hline \multirow[t]{3}{*}{ CPT1A } & rs11228368 & $\mathrm{G}(0.138)$ & $2.0 \times 10^{-13}$ & 0.0004 & $5.2 \times 10^{-16}$ & $3.3 \times 10^{-23}$ & 0.157 & $1.6 \times 10^{-16}$ \\
\hline & rs3019594 & $A(0.217)$ & $2.2 \times 10^{-27}$ & $1.1 \times 10^{-5}$ & $1.3 \times 10^{-27}$ & $6.6 \times 10^{-39}$ & 0.040 & $1.7 \times 10^{-24}$ \\
\hline & rs613084 & $A(0.065)$ & $7.2 \times 10^{-13}$ & 0.054 & $1.6 \times 10^{-9}$ & $3.2 \times 10^{-11}$ & 0.037 & $2.1 \times 10^{-7}$ \\
\hline
\end{tabular}

${ }^{*} p$-Values $<0.0002$ are considered significant after adjusting for multiple testing. These are depicted in bold.

Table 3 | Measured genotype association results for SNPs with D6D.

\begin{tabular}{|c|c|c|c|c|c|c|c|}
\hline \multirow[t]{3}{*}{ Gene } & \multirow[t]{3}{*}{ SNP } & \multicolumn{6}{|c|}{ MGA ( $p$-value)* } \\
\hline & & \multicolumn{3}{|c|}{ RBC } & \multicolumn{3}{|c|}{ Plasma } \\
\hline & & $18: 3 \omega-6$ & $18: 2 \omega-6$ & D6D & $18: 3 \omega-6$ & $18: 2 \omega-6$ & D6D \\
\hline \multirow[t]{3}{*}{ FADS1 } & rs174541 & 0.00017 & $2.3 \times 10^{-9}$ & $1.1 \times 10^{-12}$ & 0.003 & 0.003 & $2.6 \times 10^{-5}$ \\
\hline & rs174546 & 0.0005 & $1.3 \times 10^{-9}$ & $4.8 \times 10^{-12}$ & 0.004 & 0.0008 & $4.8 \times 10^{-5}$ \\
\hline & rs174556 & 0.00016 & $6.8 \times 10^{-9}$ & $2.0 \times 10^{-12}$ & 0.005 & 0.0016 & $4.0 \times 10^{-5}$ \\
\hline \multirow[t]{6}{*}{ FADS2 } & rs1535 & 0.00029 & $2.1 \times 10^{-9}$ & $3.1 \times 10^{-12}$ & 0.003 & 0.0018 & $2.6 \times 10^{-5}$ \\
\hline & rs174568 & $2.7 \times 10^{-4}$ & $2.4 \times 10^{-9}$ & $3.1 \times 10^{-12}$ & 0.003 & 0.002 & $2.6 \times 10^{-5}$ \\
\hline & rs174570 & 0.0033 & $2.8 \times 10^{-11}$ & $6.1 \times 10^{-11}$ & 0.056 & 0.002 & 0.0010 \\
\hline & rs174576 & 0.00033 & $2.2 \times 10^{-9}$ & $4.3 \times 10^{-12}$ & 0.003 & 0.0007 & $2.6 \times 10^{-5}$ \\
\hline & rs174579 & 0.232 & 0.0028 & 0.932 & 0.960 & 0.171 & 0.239 \\
\hline & rs968567 & 0.497 & 0.039 & 0.747 & 0.051 & 0.577 & 0.233 \\
\hline \multirow[t]{3}{*}{ CPT1A } & rs11228368 & 0.221 & $3.8 \times 10^{-6}$ & 0.423 & 0.860 & 0.009 & 0.181 \\
\hline & rs3019594 & 0.469 & $5.4 \times 10^{-12}$ & 0.012 & 0.560 & 0.0015 & 0.043 \\
\hline & rs613084 & 0.119 & $8.2 \times 10^{-6}$ & $6.7 \times 10^{-5}$ & 0.881 & 0.086 & 0.301 \\
\hline
\end{tabular}

${ }^{*} p$-Values $<0.0002$ are considered significant after adjusting for multiple testing. These are depicted in bold.

the metabolism of dietary PUFAs (Martinelli et al., 2009). Given the dependence of pro- or anti-inflammatory eicosanoid formation on the availability of $\omega-3$ or $\omega-6$ PUFAs, the form of dietary PUFA consumed is critical. In our study group, dietary $\omega-3$ and $\omega-6$ PUFA were found to positively associated with age, with younger individuals consuming more of saturated fatty acidrich foods indicating a shift away from traditional foods rich in $\omega$-3 FAs.

In the current study, a strong association between the SNPs and estimated plasma and erythrocyte D5D activities was observed. In particular, minor alleles of FADS1, FADS2, and CPT1A SNPs were associated with higher desaturase activity. Few studies have been conducted to examine the association of FADS1 and FADS2 SNPs with estimated desaturase activities. Polymorphisms in the FADS cluster have been found to be significantly associated with serum estimated D5D and D6D activities in European adolescents and adults from an island population of European descent (Bokor et al., 2010; Mathias et al., 2010). In contrast to our study, they found minor alleles in FADS1 and FADS2 genes to be associated with lower D5D activities. In another study, Martinelli et al. (2008) found FADS haplotypes to be associated with higher estimated erythrocyte desaturase activity. Similarly in a study conducted in Caucasian and Asian adults FADS1 and FADS2 SNPs were associated with altered desaturase activity (Merino et al., 2011). On the 
Table 4 | Measured genotype association (MGA) results for SNPs with CVD-related phenotypes.

\begin{tabular}{|c|c|c|c|c|c|c|c|c|}
\hline \multirow[t]{2}{*}{ Gene } & \multirow[t]{2}{*}{ SNP } & \multicolumn{7}{|c|}{ MGA (p-value)* } \\
\hline & & ApoA1 & Total cholesterol & Triglycerides & HDL & LDL & Heart rate & Plaque \\
\hline \multirow[t]{3}{*}{ FADS1 } & rs174541 & 0.010 & 0.623 & 0.120 & 0.0003 & 0.672 & $2.9 \times 10^{-5}$ & 0.808 \\
\hline & rs174546 & 0.011 & 0.685 & 0.192 & 0.00057 & 0.565 & $1.9 \times 10^{-5}$ & 0.856 \\
\hline & rs174556 & 0.003 & 0.455 & 0.095 & $9.0 \times 10^{-5}$ & 0.794 & $1.6 \times 10^{-5}$ & 0.775 \\
\hline \multirow[t]{6}{*}{ FADS2 } & rs1535 & 0.015 & 0.646 & 0.128 & 0.00047 & 0.665 & $1.0 \times 10^{-5}$ & 0.664 \\
\hline & rs174568 & 0.016 & 0.697 & 0.127 & 0.0006 & 0.653 & $8.5 \times 10^{-6}$ & 0.708 \\
\hline & rs174570 & 0.011 & 0.119 & 0.234 & 0.001 & 0.484 & $6.1 \times 10^{-8}$ & 0.213 \\
\hline & rs174576 & 0.010 & 0.679 & 0.114 & 0.00037 & 0.598 & $6.9 \times 10^{-6}$ & 0.679 \\
\hline & rs174579 & 0.249 & 0.930 & 0.508 & 0.097 & 0.149 & 0.0066 & 0.329 \\
\hline & rs968567 & 0.510 & 0.155 & 0.900 & 0.969 & 0.094 & 0.0063 & 0.143 \\
\hline \multirow[t]{3}{*}{ CPT1A } & rs11228368 & 0.026 & 0.289 & 0.439 & 0.057 & 0.780 & $8.6 \times 10^{-8}$ & 0.056 \\
\hline & rs3019594 & 0.001 & 0.088 & 0.794 & 0.001 & 0.729 & $2.8 \times 10^{-10}$ & 0.206 \\
\hline & rs613084 & 0.001 & 0.177 & 0.997 & $7.1 \times 10^{-5}$ & 0.670 & 0.0009 & 0.817 \\
\hline
\end{tabular}

${ }^{*} p$-Values $<0.0002$ are considered significant after adjusting for multiple testing. These are depicted in bold.

Table 5 | Bayesian quantitative trait nucleotide analysis for erythrocyte and plasma D5D.

\begin{tabular}{|c|c|c|c|c|c|c|c|}
\hline Gene & SNP & Location in the gene & Mean effect size & Posterior probability of a functional effect & $\mathbf{A A}^{\mathbf{a}}$ & $A B^{a}$ & $\mathbf{B B}^{\mathbf{a}}$ \\
\hline \multicolumn{8}{|c|}{ ERYTHROCYTE } \\
\hline FADS1 & rs174541 & Flanking $3^{\prime}$ UTR & 0.168 & 1 & $7.65(2.4)^{b}$ & $9.73(2.1)$ & $13.56(3.1)$ \\
\hline FADS2 & rs174568 & Flanking $3^{\prime}$ UTR & 0.171 & 1 & $7.65(2.4)$ & $9.75(2.1)$ & $13.56(3.1)$ \\
\hline СРТ1А & rs3019594 & Intron & 0.172 & 0.8 & $7.49(2.2)$ & $8.85(2.4)$ & $12.40(3.1)$ \\
\hline \multicolumn{8}{|c|}{ PLASMA } \\
\hline FADS1 & rs174541 & Flanking $3^{\prime}$ UTR & 0.168 & 0.5 & $0.63(0.3)$ & $0.85(0.3)$ & $1.47(0.3)$ \\
\hline FADS2 & rs174568 & Flanking $3^{\prime}$ UTR & 0.174 & 0.6 & $0.63(0.3)$ & $0.86(0.3)$ & $1.47(0.3)$ \\
\hline СРТ1А & rs3019594 & Intron & 0.261 & 1 & $0.60(0.3)$ & $0.81(0.3)$ & $1.19(0.3)$ \\
\hline
\end{tabular}

${ }^{a} A$, major allele; $B$, minor allele.

${ }^{b}$ Mean (standard deviation).

other hand, several population studies have reported associations of FADS SNPs with PUFAs. In the first of a series of studies, FADS1 and FADS2 SNPs were associated with fatty acids in phospholipids in Europeans and accounted for about $28 \%$ of the variance in fatty acids (Schaeffer et al., 2006). Minor alleles of these genotypes were associated with higher levels of LA18: $2 \omega-6$ and ALA18: $3 \omega-3$ and lower levels of AA 20:4 $\omega-6$ and EPA 20:5 $\omega$-3. This study was replicated by Rzehak et al. (2009) in a Bavarian population of Germany. In addition to phospholipids, this study also demonstrated an association between FADS1 and FADS2 SNPs and erythrocyte membrane PUFAs. Similarly, PUFAs in erythrocytes and phospholipids were associated with FADS1 and FADS2 SNPs in an Italian population (Malerba et al., 2008). In these studies, minor alleles were associated with higher ALA levels (Martinelli et al., 2008) and lower levels of AA (Malerba et al., 2008) in accordance with Schaeffer et al. (2006). However, in our study, minor alleles of all three genes were associated with increased plasma and erythrocyte AA 20:4 $\omega-6$ levels and reduced EPA 20:5 $\omega$-3 levels. The differences observed here may be attributed to the differences in population structure, or LD patterns of the SNPs based on population groups and dietary patterns.
Genetic studies conducted with PUFAs so far have found associations with SNPs on FADS1 and FADS2 but not those on CPT1A. Studies on desaturases in humans are mostly based on desaturase activity estimates calculated from product:precursor fatty acid ratios. Because most of the lipids in circulation in the fasting state are derived from the liver, these desaturation indices calculated from fasting samples are thought to reflect hepatic desaturase activity (Sjogren et al., 2008; Merino et al., 2010). Thus, the role of liver-specific CPT1A enzyme in the regulation of liver-specific desaturases, in addition to PUFAs, assumes significance. This is supported by our results, which show strong association of $C P T 1 A$ SNPs with estimated plasma and erythrocyte D5D activities.

The CPT1A gene is $60 \mathrm{~kb}$ long with 18 coding exons and is known to be modulated by dietary and hormonal factors in tissues such as heart, muscle, and liver (Gobin et al., 2002; Bonnefont et al., 2004). CPT1A is a liver-specific outer-membrane enzyme that plays a key role in the transport of PUFAs across the mitochondrial membrane and in $\beta$-oxidation. Unlike short- and medium-chain fatty acids, PUFAs cannot be transported across the mitochondrial membrane by simple diffusion. The PUFAs are first converted to their co-A esters and in combination with carnitines they are 
A

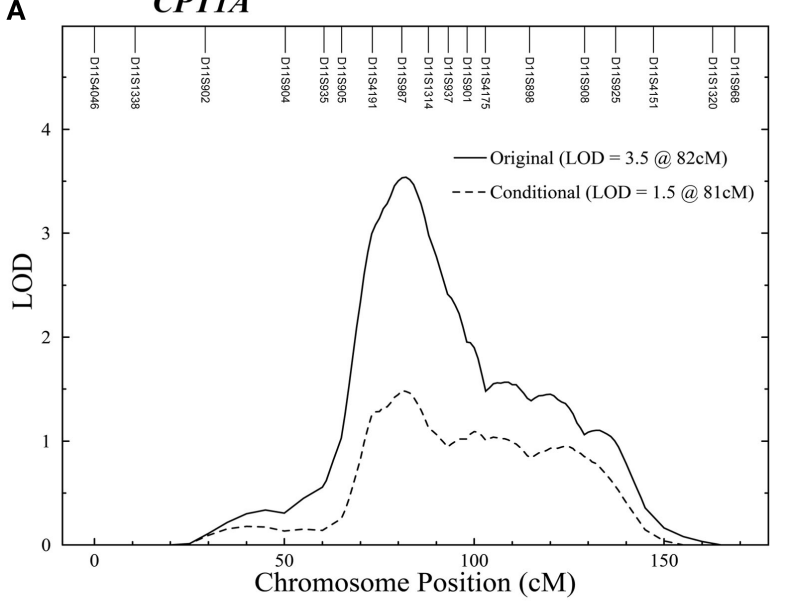

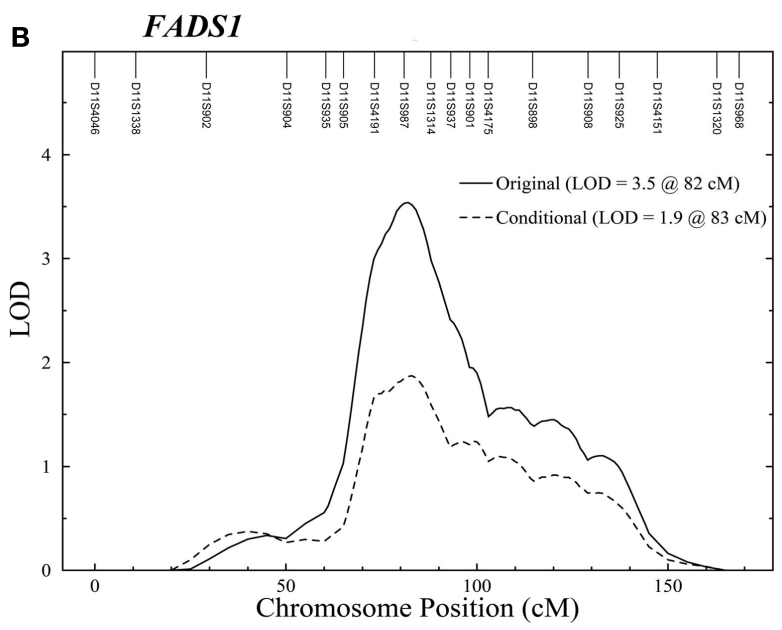

C FADS2

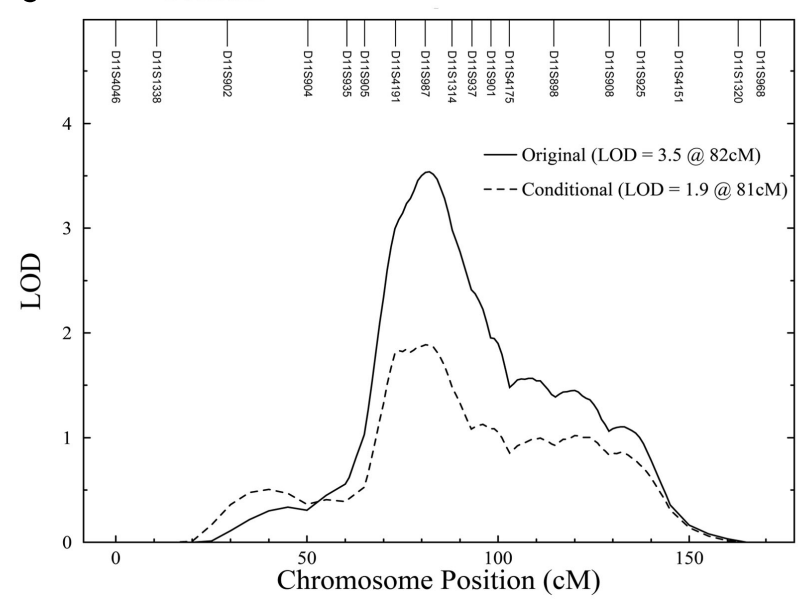

FIGURE 4 | Conditional linkage analysis for erythrocyte D5D on chromosome 11. Linkage analysis conditional on the most significantly associated SNP in (A) CPT1A, (B) FADS1, and (C) FADS2. Solid line, erythrocyte D5D; dashed line, erythrocyte D5D conditional on SNPs. converted to acylcarnitines by CPT1A enzyme and transported across the mitochondrial membrane (Prip-Buus et al., 2001). In a study conducted in a Greenland Inuit population, Rajakumar et al. (2009) found that variant P479L of CPT1A was very common and was associated with higher levels of HDL and ApoA1 and thus may be protective against atherosclerosis. In a study in Canadians, $C P T 1 A$ and $C P T 1 B$ variants were associated with obesity traits. In addition, the investigators found that the dietary intake of fat seemed to modulate these associations, particularly in individuals who were consuming high-fat diets (Robitaille et al., 2007). In another study, haplotypes of CPT1A were associated with left ventricular mass in essential hypertension (Tripodi et al., 2005). In the current study, the minor alleles of all three genes were associated positively with all CVD risk factors and with both desaturase activity estimates.

The BQTN analysis helps narrow down the variants for further molecular and functional analyses by identifying which SNP has the highest probability of being associated with the phenotype. Our BQTN findings demonstrate with a strong statistical support that SNPs in FADS1, FADS2, and CPT1A genes have either a functional role or they are in high LD with functional variants affecting D5D activities. In addition, the high genetic variance (17-26\%) for D5D activities explained by these SNPs show the importance of FADS1, FADS2, and CPT1A gene polymorphisms in the activity of D5D. Furthermore, a linkage analysis conditional on the three SNPs resulted in approximately $60 \%$ decrease in the LOD score, indicating that the SNPs accounted for a substantial part of the QTL but did not explain the linkage signal completely.

In an effort to glean further support for the association, we conducted association analyses of cis-regulated transcripts of FADS1, FADS2, and CPT1A genes with their respective SNPs in Mexican Americans. cis-regulated transcripts are defined as transcripts that contain genetic variation within their gene and regulatory regions that affect their abundance (Göring et al., 2007). We found highly significant association of FADS1, FADS2, and CPT1A transcripts with their respective SNPs. These findings in Mexican Americans support our plasma and erythrocyte D5D associations in 
Table 6 | Measured genotype association results for SNPs with CVD-related phenotypes and cis-regulated transcripts in Mexican Americans.

\begin{tabular}{|c|c|c|c|c|c|c|c|c|c|}
\hline \multirow[t]{2}{*}{ Gene } & \multirow[t]{2}{*}{ SNP } & \multicolumn{8}{|c|}{ MGA ( $p$-value)* } \\
\hline & & Transcripts & APOA1 & Total cholesterol & Triglycerides & HDL & LDL & Heart rate & Plaque \\
\hline \multirow[t]{2}{*}{ FADS1 } & rs174546 & $1.23 \times 10^{-31}$ & 0.055 & 0.044 & 0.730 & 0.058 & 0.163 & 0.039 & 0.518 \\
\hline & rs174556 & $2.53 \times 10^{-32}$ & 0.094 & 0.038 & 0.700 & 0.090 & 0.154 & 0.101 & 0.619 \\
\hline \multirow[t]{5}{*}{ FADS2 } & rs1535 & $1.89 \times 10^{-29}$ & 0.081 & 0.069 & 0.763 & 0.072 & 0.241 & 0.114 & 0.304 \\
\hline & rs174570 & $3.42 \times 10^{-7}$ & 0.168 & 0.772 & 0.710 & 0.282 & 0.709 & 0.041 & 0.461 \\
\hline & rs174576 & $4.45 \times 10^{-29}$ & 0.053 & 0.041 & 0.727 & 0.034 & 0.184 & 0.080 & 0.514 \\
\hline & rs174579 & $3.13 \times 10^{-24}$ & 0.316 & 0.580 & 0.649 & 0.406 & 0.467 & 0.943 & 0.394 \\
\hline & rs968567 & $2.57 \times 10^{-75}$ & 0.933 & 0.034 & 0.333 & 0.518 & 0.032 & 0.370 & 0.882 \\
\hline \multirow[t]{2}{*}{ CPT1A } & rs11228368 & $2.43 \times 10^{-22}$ & 0.133 & 0.506 & 0.970 & 0.186 & 0.306 & 0.632 & 0.798 \\
\hline & rs613084 & $1.14 \times 10^{-62}$ & 0.281 & 0.883 & 0.771 & 0.234 & 0.336 & 0.587 & 0.821 \\
\hline
\end{tabular}

${ }^{*} p$-Values $<0.006$ are considered significant after adjusting for multiple testing. These are depicted in bold.

Alaskan Eskimos, indicating strong support for a significant role of FADS1, FADS2, and CPT1A SNPs in D5D activity. Lack of fatty acid data in the Mexican American population limits our ability to analyze the association of D5D in plasma or erythrocytes for replication purposes but the transcript data provides valuable insight into the role of variants in the regulation of D5D. Another limitation of this study is using estimated indices from individual fatty acids as surrogate measure of desaturase activities. However, studies conducted with desaturases have used product: precursor fatty acids ratio as an estimate or a surrogate measure of desaturase activity (Martinelli et al., 2008; Bokor et al., 2010; Merino et al., 2011), and are now well accepted given the difficulties measuring in vivo activities of these enzymes in humans. In addition, we were also able to obtain strong association of SNPs in FADS1, FADS2, and CPT1A with individual fatty acid components.

In summary, we report for the first time the association of liver-specific CPT1A SNPs with liver-specific desaturases with a strong statistical support for functional effects. In addition,

\section{REFERENCES}

Allayee, H., Roth, N., and Hodis, H. N. (2009). Polyunsaturated fatty acids and cardiovascular disease: implications for nutrigenetics. J. Nutrigenet. Nutrigenomics 2, 140-148.

Almasy, L., and Blangero, J. (1998). Multipoint quantitative-trait linkage analysis in general pedigrees. Am. J. Hum. Genet. 62, 1198-1121.

Blangero, J., and Almasy, L. (1997). Multipoint oligogenic linkage analysis of quantitative traits. Genet. Epidemiol. 14, 959-964.

Blangero, J., Göring, H. H. H., Kent, J. W. Jr., Williams, J. T., Peterson, C. P., Almasy, L., and Dyer, T. D. (2005). Quantitative trait nucleotide analysis using Bayesian model selection. Hum. Biol. 77, 541-559.

Boerwinkle, E., Chakraborty, R., and Sing, C. F. (1986). The use of measured genotype information in the analysis of quantitative phenotypes

we replicate the association of FADS1 and FADS2 SNPs with desaturase activities and PUFAs in Alaskan Eskimos.

\section{ACKNOWLEDGMENTS}

We thank the participants of the GOCADAN study for their generous participation. GOCADAN is funded with support from NIH grants U01 HL64244 and MH59490 and Norton Sound Health Corporation grant HL082458. This investigation was conducted in part in facilities constructed with support from the Research Facilities Improvement Program (NCRR, NIH) under grant numbers C06 RR014578, C06 RR013556, C06 RR015456, and C06 RR017515. Transcriptional profiling and SNP typing in the SAFHS were supported by Azar/Sheppard Foundation and NIH grants MH0708143 and MH078111, respectively.

\section{SUPPLEMENTARY MATERIAL}

The Supplementary Material for this article can be found online at http://www.frontiersin.org/Applied_Genetic_Epidemiology/ abstract

Quantitative trait locus determining dietary macronutrient intakes is located on human chromosome 2p22. Am. J. Clin. Nutr. 80, 1410-1414.

Das, U. N. (2007). A defect in the inactivity of $\Delta 6$ and $\Delta 5$ desaturases may be a factor in the initiation and progression of atherosclerosis. Prostaglandins Leukot. Essent. Fatty Acids 76, 251-268.

Ebbesson, S. O., Kennish, J., Ebbesson, L., Go, O., and Yeh, J. (1999). Diabetes is related to fatty acid imbalance in Eskimos. Int. J. Circumpolar Health 58, 108-119.

Ebbesson, S. O. E., Devereux, R. B., Cole, S., Ebbesson, L. O. E., Fabsitz, R. R., Haack, K., Harris, W. S., Howard, W. H., Laston, S., Lopez-Alverenga, J. C., MacCluer, J. W., Okin, P. M., Tejero, E., Voruganti, V. S., Wenger, C. R., Howard, B. V., and Comuzzie, A. G. (2010). Heart rate is associated with red blood cell fatty acid concentration: the GOCADAN study. Am. Heart J. 159, 1020-1025.

Ebbesson, S. O. E., Laston, S., Wenger, C. R., Dyke, B., Romenesko, T., Swenson, M., Fabsitz, R. R., MacCluer, J. W., Devereux, R. B., Roman, M., Robbins, D., and Howard, B. V. (2006). Recruitment and community interactions in the GOCADAN study. Int. J. Circumpolar Health 65, 55-64.

Glaser, C., Heinrich, J., and Koletzko, B. (2010). Role of FADS1 and FADS2 polymorphisms in polyunsaturated fatty acid metabolism. Metab. Clin. Exp. 59, 993-999.

Gobin, S., Bonnefont, J. P., Prip-Buus, C., Mugnier, C., Ferrec, M., Demaugre, F., Saudubray, J. M., Rostane, H., Djouadi, F., Wilcox, W., Cederbaum, S., Haas, R., Nyhan, W. L., Green, A., Gray, G., Girard, J., and Thuillier, L. (2002). Organization of 
the human liver carnitine palmitoyltransferase 1 gene (CPT1A) and identification of novel mutations in hypoketotic hypoglycemia. Hum. Genet. 111, 179-189.

Göring, H. H. H., Curran, J. E., Johnson, M. P., Dyer, T. D., Charlesworth, J., Cole, S. A., Jowett, J. B., Abraham, L. J., Rainwater, D. L., Comuzzie, A. G., Mahaney, M. C., Almasy, L., MacCluer, J. W., Kissebah, A. H., Collier, G. R., Moses, E. K., and Blangero, J. (2007). Discovery of expression QTLs using large-scale transcriptional profiling in human lymphocytes. Nat. Genet. 39, 1208-1216.

Harris, W., Mozaffarian, D., Rimm, E., Kris-Etherton, P., Rudel, L. L., Appel, L. J., Engler, M. M., Engler, M. B., and Sacks, F. (2009). Omega-6 fatty acids and risk for cardiovascular disease. Circulation 119, 902-907.

Heath, S. C. (1997). Markov chain Monte Carlo segregation and linkage analysis for oligogenic models. Am. J. Hum. Genet. 61, 748-760.

Howard, B. V., Devereux, R. B., Cole, S. A., Davidson, M., Dyke, B., Ebbesson, S. O., Epstein, S. E., Robinson, D. R., Jarvis, B., Kaufman, D. J., Laston, S., MacCluer, J. W., Okin, P. M., Roman, M. J., Romenesko, T., Ruotolo, G., Swenson, M., Wenger, C. R., Williams-Blangero, S., Zhu, J., Saccheus, C., Fabsitz, R. R., and Robbins, D. C. (2005). A genetic and epidemiologic study of cardiovascular disease in Alaska Natives (GOCADAN): design and methods. Int. J. Circumpolar Health 64, 206-221.

Kong, A., Gudjartsson, D. F., Sainz, J., Jonsdottir, G. M., Gudjonsson, S. A., Richardsson, B., Sjgurdardottir, S., Barnard, J., Hallbeck, B., Masson, G., Shlien, A., Palsson, S. T., Frigge, M. L., Thorgeirsson, T. E., Gulcher, J. R., and Stefansson, K. (2002). A high-resolution recombination map of the human genome. Nat. Genet. 31, 241-247.

Lattka, E., Ilig, T., Koletzko, B., and Heinrich, J. (2010). Genetic variants of the FADS1 FADS2 gene cluster as related to essential fatty acid metabolism. Curr. Opin. Lipidol. 21, 64-69.

Lattka, E., Illig, T., Heinrich, J., and Koletzko, B. (2009). FADS gene cluster polymorphisms: important modulators of fatty acid levels and their impact on atopic diseases. J. Nutrigenet. Nutrigenomics 2 , 119-128.

Malerba, G., Schaeffer, L., Xumerle, L., Klopp, N., Trabetti, E., Biscuola, M.,
Cavallari, U., Galavotti, R., Martinelli, N., Guarini, P., Girelli, D., Olivieri, O., Corrocher, R., Heinrich, J., Pignatti, P. F., and Illig, T. (2008). SNPs of the FADS gene cluster are associated with polyunsaturated fatty acids in a cohort of patients with cardiovascular disease. Lipids 43, 289-299.

Martinelli, M., Consoli, L., and Olivieri, O. (2009). A 'desaturase hypothesis' for atherosclerosis: Janus-faced enzymes in $\omega-6$ and $\omega-3$ polyunsaturated fatty acid metabolism. J. Nutrigenet. Nutrigenomics 2, 129-139.

Martinelli, N., Girelli, D., Malerba, G., Guarini, P., Illig, T., Trabetti, E., Sandri, M., Friso, S., Pizzolo, F., Schaeffer, L., Heinrich, J., Pignatti, P. F., Corrocher, R., and Olivieri, O. (2008). FADS genotypes and desaturase activity estimated by the ratio of arachidonic acid to linoleic acid are associated with inflammation and coronary artery disease. Am. J. Clin. Nutr. 88, 941-949.

Mathias, R., Vergara, C., Gao, L., Rafaels, N., Hand, T., Campbell, M., Bickel, C., Ivester, P., Sergeant, S., Barnes, K. C., and Chilton, F. H. (2010). FADS genetic variants and omega- 6 polyunsaturated fatty acid metabolism in a homogenous island population. J. Lipid Res. 51, 2766-2774.

Merino, D. M., Johnston, H., Clarke, S., Roke, K., Nielsen, D., Badawi, A., El-Sohemy, A., Ma, D. W., Mutch, D. M. (2011). Polymorphisms in FADS1 and FADS2 alter desaturase activity in young Caucasian and Asian adults. Mol. Genet. Metab. 103, 171-178.

Merino, D. M., Ma, D. W. L., and Mutch, D. M. (2010). Genetic variation in lipid desaturases and its impact on the development of human disease. Lipids Health Dis. 9, 63.

Mozaffarian, D., Micha, R., and Wallace, S. (2010). Effects of coronary heart disease of increasing polyunsaturated fat in place of saturated fat: a systematic review and meta analysis of randomized controlled trials. PLoS Med. 7, e1000252. doi:10.1371/journal.pmed.1000252

Prip-Buus, C., Thuillier, L., Abadi, N., Prasad, C., Dilling, L., Klasing, J., Demaugre, F., Greenberg, C. R., Hawarth, J. C., Droin, V., Kadhom, N., Gobin, S., Kamoun, P., Girard, J., and Bonnefont, J. P. (2001). Molecular and enzymatic characterization of a unique carnitine palmitoyltransferase $1 \mathrm{~A}$ mutation in the Jutterite community. Mol. Genet. Metab. 73, 46-54.
Rajakumar, C., Ban, M. R., Cao, H., Young, T. K., Bjerregaard, P., and Hegele, R. A. (2009). Carnitine palmitoyltransferase IA polymorphism P479L is common in Greenland Inuit and is associated with elevated plasma apolipoprotein A-1. J. Lipid Res. 50, 1223-1228.

Robitaille, J., Houde, A., Lemieux, S., Perusse, L., Gaudet, D., and Vohl, M. C. (2007). Variants within the muscle and liver isoforms of the carnitine palmitoyltransferase 1 (CPT1) gene interact with fat intake to modulate indices of obesity in French-Canadians. J. Mol. Med. 85, 129-137.

Rzehak, P., Heinrich, J., Klopp, N., Schaeffer, L., Hoff, S., Wolfram, G., Illig, T., and Linseisen, J. (2009). Evidence for an association between genetic variants of the fatty acid desaturase 1 fatty acid desaturase 2 (FADS1 FADS2) gene clutser and the fatty acid decomposition of erythrocyte membranes. Br. J. Nutr. 101, 20-26.

Schaeffer, L., Gohlke, H., Muller, M. Heid, I. M., Palmer, J., Kompauer, I., Demmelmair, H., Illig, T., Koletzko, B., and Heinrich, J. (2006). Common genetic variants of the FADS1 FADS2 gene cluster and their reconstructed haplotypes are associated with the fatty acid composition in phospholipids. Hum. Mol. Genet. 15, 1745-1756.

Sjogren, P., Sierra-Johnson, J., Gertow, K., Rosell, M., Vessby, B., de Faire, U., Hamsten, A., Hellenius, M.-L., and Fisher, R. M. (2008). Fatty acid desaturases in human adipose tissue: relationships between gene expression, desaturation indexes and insulin resistance. Diabetologia 51, 328-335.

Sobel, E., and Lange, K. (1996). Descent graphs in pedigree analysis: applications to haplotyping, location scores, and marker sharing statistics. Am. J. Hum. Genet. 58, 1323-1337.

Sun, L., Cox, N. J., and McPeek, M. S (2002). A statistical method for identification of polymorphisms that explain a linkage result. Am. J. Hum. Genet. 70, 399-411.

Tanaka, T., Shen, J., Abecasis, G. R., Kisialiou, A., Ordovas, J. M., Guralnik, J. M., Singleton, A., Bandinelli, S., Cherubin, A., Arnett, D., Tsai, M. Y., and Ferrucci, L. (2009). Genome-wide association study of plasma polyunsaturated fatty acids in the inCHIANTI study. PLoS Genet. 5, e1000338. doi:10.1371/journal.pgen.1000338
Tripodi, G., Modica, R., Stella, A., Brigatti, G., and Stella, P. (2005). Haplotype analysis of carnitine transporters and left ventricular mass in human essential hypertension. J. Ren. Nutr. 15, 2-7.

Voruganti, V. S., Cole, S. A., Ebbesson, S. O., Göring, H. H., Haack, K., Laston, S., Wenger, C. R., Tejero, M. E., Devereux, R. B., Fabsitz, R. R., MacCluer, J. W., Umans, J. G., Howard, B. V., and Comuzzie, A. G. (2010). Genetic variation in APOJ, LPL, and TNFRSF10B affects the plasma fatty acid distribution in Alaskan Eskimos. Am. J. Clin. Nutr. 91, 1574-1583.

Warensjö, E., Sundström, J., Vessby, B., Cederholm, T., and Risérus, U. (2008). Markers of dietary fat quality and fatty acid desaturation as predictors of total and cardiovascular mortality: a population-based prospective study. Am. J. Clin. Nutr. 88, 203-209.

Conflict of Interest Statement: The authors declare that the research was conducted in the absence of any commercial or financial relationships that could be construed as a potential conflict of interest.

Received: 03 March 2012; paper pending published: 02 April 2012; accepted: 30 April 2012; published online: 11 June 2012.

Citation: Voruganti VS, Higgins PB, Ebbesson SOE, Kennish J, Göring HHH, Haack K, Laston S, Drigalenko E, Wenger $C R$, Harris WS, Fabsitz RR, Devereux RB, MacCluer JW, Curran JE, Carless MA, Johnson MP, Moses EK, Blangero $J$, Umans JG, Howard BV, Cole SA and Comuzzie AG (2012) Variants in CPT1A, FADS1, and FADS2 are associated with higher levels of estimated plasma and erythrocyte delta-5 desaturases in Alaskan Eskimos. Front. Gene. 3:86. doi: 10.3389/fgene.2012.00086 This article was submitted to Frontiers in Applied Genetic Epidemiology, a specialty of Frontiers in Genetics.

Copyright (C) 2012 Voruganti, Higgins, Ebbesson, Kennish, Göring, Haack, Laston, Drigalenko, Wenger, Harris, Fabsitz, Devereux, MacCluer, Curran, Carless, Johnson, Moses, Blangero, Umans, Howard, Cole and Comuzzie. This is an open-access article distributed under the terms of the Creative Commons Attribution Non Commercial License, which permits non-commercial use, distribution, and reproduction in other forums, provided the original authors and source are credited. 